\title{
Portals of discovery
}

\author{
Assumptions, educated guesses and intuition are often unavoidably involved in the study of new \\ phenomena, and scientists may therefore make mistakes at the outset. However, this is part of the \\ research process and sometimes brilliant mistakes can lead to unexpected discoveries.
}

In his science-fiction novel A Journey to the Centre of the Earth, Jules Verne wrote, "Science, my lad, is made up of mistakes, but they are mistakes which it is useful to make, because they lead little by little to the truth." Scientists in any field would relate, in one way or another, to this thought.

The most exciting times in a researcher's career take place when a new concept or technology is introduced. Ideas proposed are always new and exciting, as unchartered territories are being explored. Results obtained are always intriguing, if not groundbreaking. They could subsequently open new research paths, attracting funding and gathering public attention. It is therefore, needless to say, gratifying to be one of the pioneers in the field.

However, it is at the same time the most challenging and error-prone period for a researcher. The reason is simply that first observations often involve initial interpretations and hypotheses. Explaining a new phenomenon requires a very deep understanding of the topic. Rigorous studies and precise procedures are needed before well-informed conclusions can be made and research findings published. This is especially difficult at the initial stage because scientists find themselves searching in the dark, with too many pieces missing from the puzzle, and with limited methodology and data with which to elucidate the science. In the early stages, inevitably, assumptions, educated guesses and intuition are often relied on. They are crucial in advancing science, yet are hazardous, because erroneous or wrongly interpreted data may hinder or stall progress by misinforming scientists.

Nevertheless, making scientific mistakes is not entirely a negative thing. "Mistakes are our portals of discovery," said the Irish novelist and poet, James Joyce. Indeed, errors and failed experiments, although disappointing, provide an alternative way of advancing research by ruling out impossible approaches, interpretations and analyses, stimulating discussion, and sparking scientific enquiries and new ideas.

The history of human knowledge is full of examples of great philosophers and scientists making equally great mistakes.

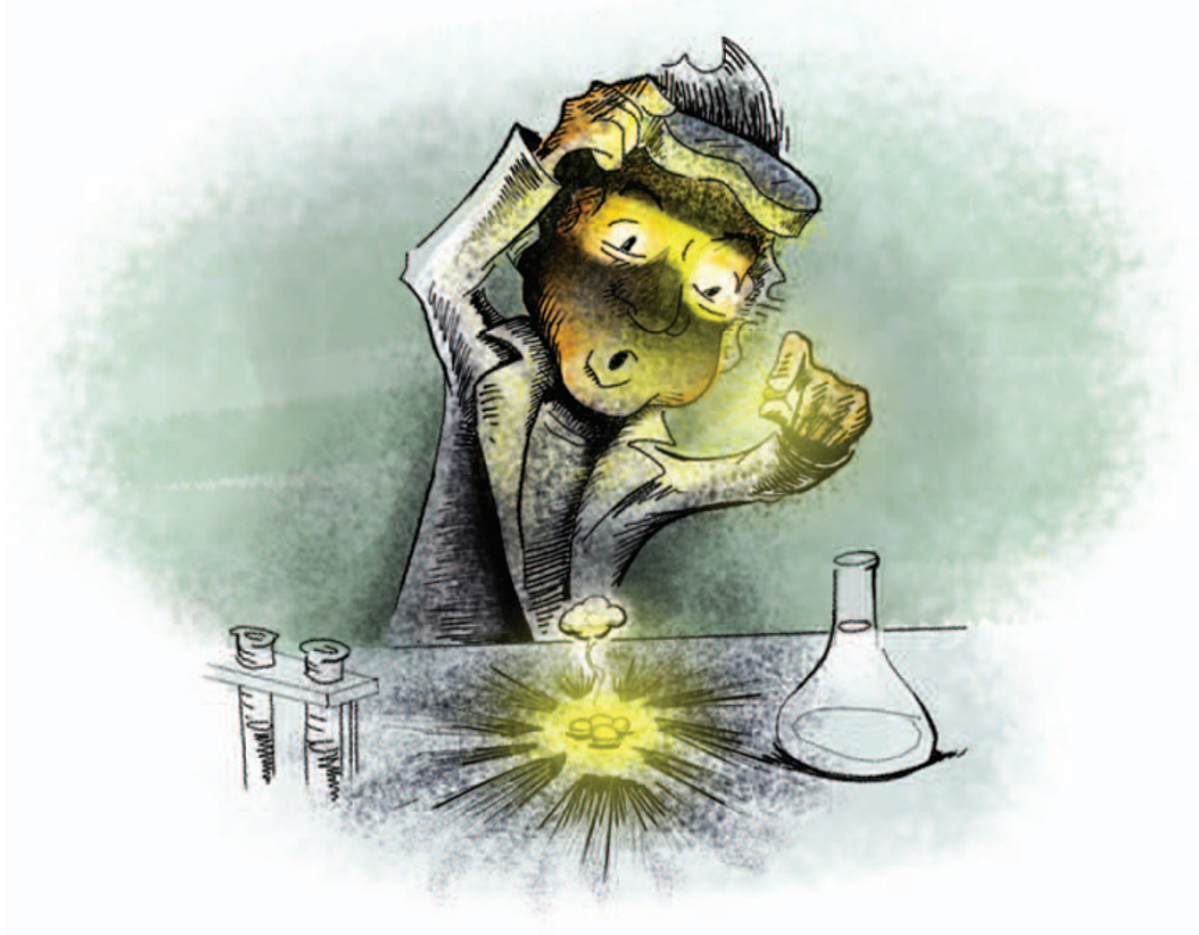

A good classical example is Aristotle's idea that heavy objects fall more rapidly than light objects. Although this was indeed a mistake, Aristotle taught us how to use fresh observations and additional information to correct our mistakes. Another example is the atomic model proposed by Niels Bohr in the 1910s. Bohr created a model of the atom that, as we know now, was not completely right, yet it inspired new thoughts on quantum mechanics. A recent example is physicist Nick Herbert's 1981 investigation of strange features of quantum mechanics for a scheme that could send signals faster than the speed of light. The efforts to uncover its subtle flaw prompted a new understanding of quantum information theory ${ }^{1}$.

In September 2011, the OPERA experiments reported that neutrinos travelled $0.002 \%$ faster than light, violating Einstein's special theory of relativity. This groundbreaking finding was later disproved by different teams of physicists. "The story captured the public imagination, and has given people the opportunity to see the scientific method in action - an unexpected result was put up for scrutiny, thoroughly investigated and resolved in part thanks to collaboration between normally competing experiments. That's how science moves forward," said Sergio Bertolucci, research director at CERN, at the 25th International Conference on Neutrino Physics and Astrophysics in Kyoto, Japan, in June $2012^{2}$. Although this seems like disappointing news now, we have yet to witness its impact on future science. Only time will tell.

Mistakes are as unavoidable in science as they are in life generally, and should not be frowned on too harshly. What is important is to embrace the learning potential of mistakes as part of the research process. Furthermore, scientists must always be alert not to overlook what could be a brilliant mistake that leads to an altogether unexpected discovery.

\section{References}

1. Kaiser, D. \& Creager, A. N. H. Sci. Am. 306, 70-75 (2012).

2. http://press.web.cern.ch/press/PressReleases/Releases2011/ PR19.11E.html 